\title{
The reliability of suicide statistics: a systematic review
}

Ingvild Maria Tøllefsen ${ }^{1,2^{*}}$, Erlend Hem² and Øivind Ekeberg ${ }^{1,2}$

\begin{abstract}
Background: Reliable suicide statistics are a prerequisite for suicide monitoring and prevention. The aim of this study was to assess the reliability of suicide statistics through a systematic review of the international literature.

Methods: We searched for relevant publications in EMBASE, Ovid Medline, PubMed, PsycINFO and the Cochrane Library up to October 2010. In addition, we screened related studies and reference lists of identified studies. We included studies published in English, German, French, Spanish, Norwegian, Swedish and Danish that assessed the reliability of suicide statistics. We excluded case reports, editorials, letters, comments, abstracts and statistical analyses. All three authors independently screened the abstracts, and then the relevant full-text articles.

Disagreements were resolved through consensus.

Results: The primary search yielded 127 potential studies, of which 31 studies met the inclusion criteria and were included in the final review. The included studies were published between 1963 and 2009. Twenty were from Europe, seven from North America, two from Asia and two from Oceania. The manner of death had been reevaluated in 23 studies (40-3,993 cases), and there were six registry studies (195-17,412 cases) and two combined registry and re-evaluation studies. The study conclusions varied, from findings of fairly reliable to poor suicide statistics. Thirteen studies reported fairly reliable suicide statistics or under-reporting of 0-10\%. Of the 31 studies during the 46 -year period, 52\% found more than 10\% under-reporting, and 39\% found more than 30\% underreporting or poor suicide statistics. Eleven studies reassessed a nationwide representative sample, although these samples were limited to suicide within subgroups. Only two studies compared data from two countries.

Conclusions: The main finding was that there is a lack of systematic assessment of the reliability of suicide statistics. Few studies have been done, and few countries have been covered. The findings support the general under-reporting of suicide. In particular, nationwide studies and comparisons between countries are lacking.
\end{abstract}

\section{Background}

In recent decades, research on suicide and suicidal behaviour has expanded. Preventing suicide and reducing suicidal behaviour are important targets of the World Health Organization (WHO) [1]. The WHO has estimated that, worldwide, about one million people die by suicide every year, representing a global annual suicide rate of 16 per 100,000 people [2]. In addition, the suicide attempt rate is about $10-15$ times more frequent than the suicide rate $[3,4]$. These suicide estimates are based on national mortality statistics, with suicide rates ranging from no suicides per 100,000 people per year in

\footnotetext{
* Correspondence: i.m.tollefsen@medisin.uio.no

'Department of Acute Medicine, Oslo University Hospital Ullevaal, PO Box 4956, Nydalen NO-0424 Oslo, Norway

Full list of author information is available at the end of the article
}

countries such as Egypt, Haiti and Honduras, to more than 30 suicides per 100,000 people per year in Belarus, the Russian Federation and Lithuania [5].

Most countries in the industrialized world started to register the cause and manner of deaths at the end of the 19th or the beginning of the 20th century. WHO member states use the International Classification of Diseases (ICD) to classify diseases and death certificates. The first edition, known as the International List of Causes of Death, was adopted in 1893. Even with this long tradition of classification, it is difficult to compare statistics between countries and periods because of differences between countries in methods of classification and registration, and because the manner of registration has changed over time.

\section{Biomed Central}

() 2012 Tøllefsen et al; licensee BioMed Central Ltd. This is an Open Access article distributed under the terms of the Creative Commons Attribution License (http://creativecommons.org/licenses/by/2.0), which permits unrestricted use, distribution, and reproduction in any medium, provided the original work is properly cited. 
National mortality registers have been used in the past few decades for surveillance and research on suicide, and can be used to examine the effects of preventive strategies and priorities in health policy. Epidemiological or socio-demographic theories about suicide and the effects of intervention depend on reliable suicide statistics. Many scientists have pointed out this challenge $[6,7]$, but to our knowledge, no systematic research has been done in this field.

The aim of this study was to assess the reliability of suicide statistics through a systematic review of the international literature.

\section{Methods}

\section{Search strategy}

The first author (IMT) searched for relevant literature up to June 2009 in five databases: EMBASE (from 1980), Ovid Medline (from 1950), PsycINFO (from 1806), the Cochrane Library (from 1993) and PubMed (from 1950). The search strategy included subject headings/MeSH terms and free text. MeSH headings and free text included the terms "suicide" combined with "reliability", "test reliability", "validity", "test validity", "reproducibility", "reproducibility of results", "cause of death" and "death certificates". The search was restricted to humans. The search was not restricted by language, publication type or study design (Additional file 1). In addition, related studies and reference lists of identified studies were screened. Update searches were performed in October 2010, but no new studies were found.

\section{Study selection}

All abstracts identified using the above search strategy were reviewed. The first author (IMT) excluded studies that were obviously irrelevant to this review. Then, the three authors screened the abstracts for relevancy, and independently reviewed the abstracts of all potentially relevant studies. Studies were included if they met the inclusion criteria of having the aim of studying the reliability of suicide statistics, and being published in English, German, French, Spanish, Norwegian, Swedish or Danish.

Studies were excluded if they were case reports, editorials, letters, comments, statistical analyses or studies only presented as abstracts. Any disagreements or differences in the extracted data between authors were resolved through consensus. If there were any doubts, we included the abstract and read the full text of the article. After excluding articles based on the abstracts, the authors performed a second, stricter screening by examining full-text reports of the remaining records. Disagreements regarding the eligibility were resolved through consensus. Reasons for exclusion were documented. We included all studies on the reliability of suicide statistics. The process of study inclusion is shown in Figure 1.

We did not attempt a meta-analysis because of methodological differences across studies. We assessed the methodological quality of the included studies using six criteria; area studied, population studied, cause and manner of death studied, how the reliability were assessed, the information the re-evaluations were based on, and number of cases included. The criteria of assessment of methodological quality are shown in Figure 2.

Three ways of assessing the reliability of suicide statistics There are three ways to assess the reliability of suicide statistics: re-evaluation studies, registry studies and statistical analyses. Re-evaluation studies are studies where the manner and cause of death were re-evaluated. Registry studies are studies where two cause-of-death registers are compared. Statistical analyses are studies where the suicide rate is calculated by adding other categories of manner and cause of death, usually undetermined deaths, open verdicts, and unintentional poisoning and drowning. We excluded statistical analyses from this review because the choice of which other categories of manner or cause of death to include often relies on registry or re-evaluation studies.

\section{Analyses}

Some studies did not calculate the percentage of underreporting, but calculated a study suicide rate and compared it with the official suicide rate. In those studies in which it was possible to estimate the percentage of under-reporting, we calculated the percentage by dividing the difference between the study and official suicide rates by the official suicide rate (under-reporting = (study suicide rate-official suicide rate)/official suicide rate).

\section{Results}

\section{Study selection}

The primary search yielded 127 potential studies. Of these, 31 studies [8-38] met the inclusion criteria, with a population of 46,401 cases in the final review. Three of the studies did not describe the exact number of cases, and were excluded from the total number of cases. Of the 96 excluded articles, 76 were excluded because they did not study the reliability of suicide statistics, 12 were statistical analyses, two were letters, one was a comment and five were excluded because of language (Romanian, Portuguese, Czech, Serbian, Dutch).

\section{Study characteristics \\ Methodologies and sample size}

Of the 31 included studies, 23 were re-evaluation studies, with a total population of 11,795 cases (range: 40 - 


\begin{tabular}{ll}
\hline EMBASE & 52 \\
MEDLINE & 624 \\
PsycINFO & 438 \\
Cochrane & 33 \\
PubMed & 615 \\
Total & 1762
\end{tabular}

Articles excluded on basis of abstract through references provided from the extracted articles and through related articles.

95 articles identified through literature search.

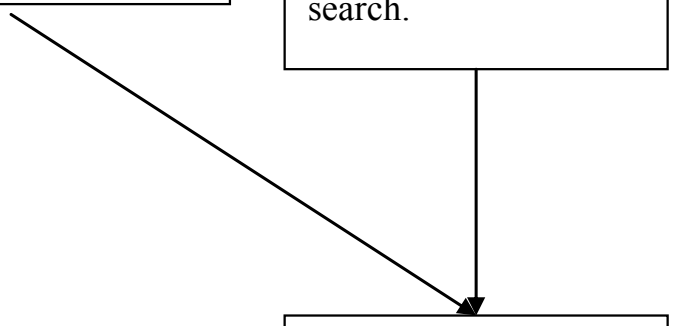

127 abstracts screened

62 articles excluded

- 54 studies did not study the reliability of suicide statistics - 5 studies excluded because of language -2 letters 65 articles obtained in

-1 comment full text

34 articles excluded

- 22 studies did not study the reliability of suicide statistics

31 articles included

- 12 were statistical analysis

Figure 1 Study selection flow chart

$3,993)$. Six studies were registry studies, with a total population of 31,436 cases (range: 195-17,412). The remaining two studies were combined registry and reevaluation studies, with a total population of 3,170 cases
(442 and 2,728 cases). Eight re-evaluation studies evaluated a nationwide representative sample, although these samples were limited to suicide within subgroups. Three registry studies evaluated a nationwide sample. Only 


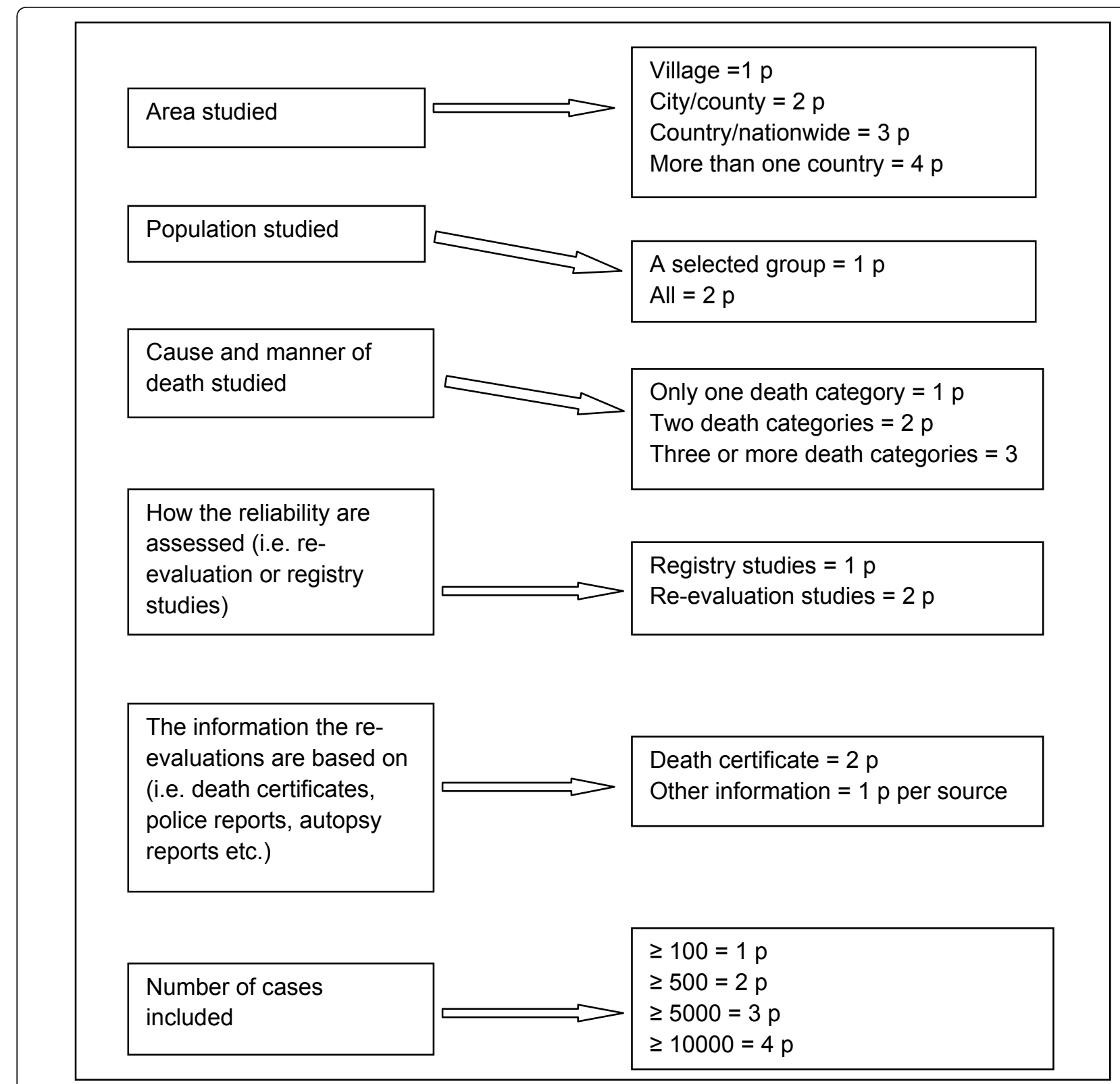

Figure 2 Assessment of methodological quality.

two studies compared data from two or more countries. Characteristics of the included studies are presented in Table 1.

\section{Year and location of studies}

The included studies were published between 1963 and 2009. Fourteen studies were published between 1963 and 1989, ten between 1990 and 1999 and seven after 2000. Twenty were from Europe, seven from North America, two from Asia, and two from Oceania.

\section{Characteristics of the study population}

Of the included studies, one re-evaluated the reliability of suicide statistics within the military system. Two studies examined the causes of death in cohorts: one of young males conscripted for military service and one of twins. The other studies included all deaths within defined time periods, locations or subgroups according to the manner of death. Some studies evaluated only suicides, whereas others included homicides, accidents and undetermined deaths.

\section{Analysis of the included studies}

The main conclusions of the studies varied, with findings ranging from fairly reliable suicide statistics to considerable under-reporting. Thirteen studies (42\%) reported fairly reliable suicide statistics or under- 
Table 1 Characteristics of the included studies by type of study and publication year

\begin{tabular}{|c|c|c|c|c|c|c|c|c|}
\hline Study & $\begin{array}{l}\text { Publication- } \\
\text { year }\end{array}$ & $\begin{array}{l}\text { Study } \\
\text { period }\end{array}$ & Area studied & Population & $\begin{array}{l}\text { Cause and } \\
\text { manner of } \\
\text { death } \\
\text { studied }\end{array}$ & $\begin{array}{l}\text { Method of } \\
\text { sampling }\end{array}$ & $\begin{array}{l}\text { Number } \\
\text { of cases } \\
\text { (n) }\end{array}$ & Results \\
\hline \multicolumn{9}{|c|}{ Re-evaluation studies } \\
\hline $\begin{array}{l}\text { Tsung- } \\
\text { Hsueh, Lu } \\
\text { et al. [37] }\end{array}$ & 2006 & $\begin{array}{l}1.1- \\
31.12 .2002\end{array}$ & $\begin{array}{l}\text { Taiwan } \\
\text { (nationwide) }\end{array}$ & All & $S, U$ & Re-evaluated DC & 3993 & $\begin{array}{l}\text { Fair quality of mode of } \\
\text { death certification (MOD). }\end{array}$ \\
\hline $\begin{array}{l}\text { Carr J.R. et } \\
\text { al. [36] }\end{array}$ & 2004 & 1998-1999 & USA (nationwide) & US Military & $\begin{array}{l}A^{*}, U, H \text { and } \\
\text { deaths } \\
\text { occurred } \\
\text { within } 30 \\
\text { days of } \\
\text { retirement }\end{array}$ & $\begin{array}{l}\text { Re-evaluated } \\
D C, A R, T R, C R \text {, } \\
M J \text { and } \\
\text { investigative } \\
\text { agency reports }\end{array}$ & 1844 & $\begin{array}{l}\text { Concluded } 21 \% \\
\text { underestimation. }\end{array}$ \\
\hline
\end{tabular}

Found $17 \%$ underreporting, and additional $4 \%$ of deaths that were suspicious for suicide.

\begin{tabular}{|c|c|c|c|c|c|c|c|c|}
\hline $\begin{array}{l}\text { Ahlm, K. et } \\
\text { al. [33] }\end{array}$ & 2001 & 1999 & $\begin{array}{l}\text { Sweden } \\
\text { (nationwide) }\end{array}$ & All & Traffic deaths & $\begin{array}{l}\text { Re-evaluated } \\
\text { AR, DC, MJ and } \\
\text { PR }\end{array}$ & 580 & I $1.5 \%$ under-reporting. \\
\hline
\end{tabular}

\begin{tabular}{|c|c|c|c|c|c|c|c|c|}
\hline & & & & & & & & $\begin{array}{l}\text { Found } 3 \% \text { ( } 18 \text { of } 580 \text { ) } \\
\text { suicides among the officially } \\
\text { registered accidental traffic } \\
\text { deaths. }\end{array}$ \\
\hline $\begin{array}{l}\text { Sampson, } \\
\text { H.M. and } \\
\text { Rutty, G.N } \\
{[31]}\end{array}$ & 1999 & 1992-1997 & $\begin{array}{l}\text { South Yorkshire } \\
\text { (West), England }\end{array}$ & All & $\mathrm{S}, \mathrm{OV}$ & $\begin{array}{l}\text { Re-evaluated } \\
M J \text {, AR, and SN }\end{array}$ & 295 & $\begin{array}{l}\text { Official national data may } \\
\text { under-report the annual } \\
\text { suicide rate by over } 20 \% \text {. }\end{array}$ \\
\hline $\begin{array}{l}\text { Ohberg, A. } \\
\text { et al. [30] }\end{array}$ & 1998 & $\begin{array}{l}1.4 .1987- \\
31.3 .1988\end{array}$ & $\begin{array}{l}\text { Finland } \\
\text { (nationwide) }\end{array}$ & All & $U$ & $\begin{array}{l}\text { Re-evaluated MJ } \\
\text { and } \\
\text { psychological } \\
\text { autopsies. }\end{array}$ & 139 & $\begin{array}{l}\text { Undetermined deaths } \\
\text { resembled suicides and } \\
\text { appeared to reduce the } \\
\text { suicide rate by } 10 \%\end{array}$ \\
\hline $\begin{array}{l}\text { Connolly, J. } \\
\text { F. and } \\
\text { Cullen, A } \\
{[28]}\end{array}$ & 1995 & 1978-1992 & $\begin{array}{l}\text { County Mayo, } \\
\text { Ireland }\end{array}$ & All & $S, A^{* *}, U$ & $\begin{array}{l}\text { Re-evaluated } \\
\text { DC, MJ }\end{array}$ & 220 & $\begin{array}{l}35 \% \text { deaths were miscoded } \\
(n=56) \text { or unregistered ( } n \\
=16) .\end{array}$ \\
\hline $\begin{array}{l}\text { O'Donnell, } \\
\text { l. and } \\
\text { Farmer, R } \\
{[29]}\end{array}$ & 1995 & $\begin{array}{l}\text { A 5-year } \\
\text { study } \\
\text { period }\end{array}$ & London (England) & All & $\begin{array}{l}\mathrm{S} \text { (deaths on } \\
\text { the London } \\
\text { Underground } \\
\text { railway } \\
\text { system) }\end{array}$ & $\begin{array}{l}\text { Re-evaluated. } \\
\text { Records from } \\
\text { LUL and the } \\
\text { British Transport } \\
\text { Police. } \\
\text { Prospective } \\
\text { design }\end{array}$ & 242 & $\begin{array}{l}\text { Substantial underestimation } \\
\text { of the true number of } \\
\text { suicides. }\end{array}$ \\
\hline
\end{tabular}

\begin{tabular}{|c|c|c|c|c|c|c|c|c|}
\hline & & & & & & & & $\begin{array}{l}\text { Verdicts other than suicide } \\
\text { were returned on } 25 \% \text { and } \\
50 \% \text { of the women and } \\
\text { men, respectively. }\end{array}$ \\
\hline \multirow[t]{2}{*}{$\begin{array}{l}\text { Scott, K.W. } \\
\text { M [26] }\end{array}$} & 1994 & 1976-1990 & $\begin{array}{l}\text { The borough of } \\
\text { Wolverhampton } \\
\text { (250.566) } \\
\text { (England) }\end{array}$ & All & S & Re-evaluated AR & 394 & ๆ $69 \%$ under-reporting. \\
\hline & & & & & & & & $\begin{array}{l}\text { Estimated suicide rate of } \\
10.5 / 100,000 \text { per year. The } \\
\text { official suicide rate were } \\
6.2 / 100000 \text { per year. The } \\
\text { coroners only reflected } 59 \% \\
\text { of the probable true suicide } \\
\text { rate. }\end{array}$ \\
\hline $\begin{array}{l}\text { Allebeck, P. } \\
\text { et al. [24] }\end{array}$ & 1991 & 1969-1983 & $\begin{array}{l}\text { Sweden } \\
\text { (nationwide) }\end{array}$ & $\begin{array}{l}\text { Males } \\
\text { conscripted } \\
\text { for military } \\
\text { service in } \\
1969-70\end{array}$ & $\mathrm{CO}, \mathrm{S}, \mathrm{U}, \mathrm{TD}$ & $\begin{array}{l}\text { Re-evaluated } \\
\text { DC, PR, MJ, TR } \\
\text { and AR in a } \\
\text { cohort of } 50.465\end{array}$ & 322 & $\begin{array}{l}\text { High accuracy. Findings } \\
\text { indicate an underreporting } \\
\text { of suicide in cases of } \\
\text { poisoning and other } \\
\text { undetermined cases. }\end{array}$ \\
\hline
\end{tabular}


Table 1 Characteristics of the included studies by type of study and publication year (Continued)

\begin{tabular}{|c|c|c|c|c|c|c|c|c|}
\hline $\begin{array}{l}\text { Rodriguez- } \\
\text { Pulido, F. et } \\
\text { al. [23] }\end{array}$ & 1991 & 1977-1983 & $\begin{array}{l}\text { Canary Islands } \\
\text { (Spain) }\end{array}$ & All & VD, ND & $\begin{array}{l}\text { Re-evaluated } \\
\text { AR, SN, TE and } \\
\text { JP }\end{array}$ & 775 & ११ 104\% under-reporting. \\
\hline
\end{tabular}
al. [23]

Lack of validity and reliability of official figures of suicide. Recorded 8.1/ 100,000 suicides per year. The official published statistics recorded 3.98/ 100,000 per year for the same period. This represents $49 \%$ of which was found in this study.

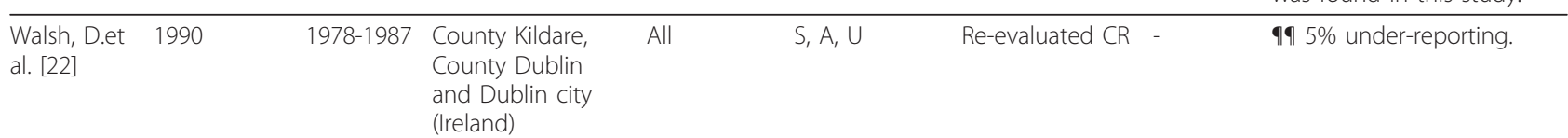

Reflecting the suicide rate accurately.

Estimated 5.9/100,000 suicides per year. The official suicide rate were 5.6/100,000.

\begin{tabular}{|c|c|c|c|c|c|c|c|c|}
\hline $\begin{array}{l}\text { Huusko, R. } \\
\text { and } \\
\text { Hirvonen, J } \\
\text { [20] }\end{array}$ & 1988 & 1981 & Oulu, Finland & All & $S, A, U$ & $\begin{array}{l}\text { Re-evaluated PR, } \\
A R \text { and } M J\end{array}$ & 283 & $\begin{array}{l}\text { The official figure for } \\
\text { suicides could be as much } \\
\text { as } 18.9 \% \text { too low. }\end{array}$ \\
\hline $\begin{array}{l}\text { Ekeberg, } \varnothing \text {. } \\
\text { et al. [17] }\end{array}$ & 1985 & $\begin{array}{l}24.5 .1978 \\
- \\
26.4 .1981\end{array}$ & $\begin{array}{l}\text { Norway (except } \\
\text { Hordaland and } \\
\text { Sogn og fjordane) }\end{array}$ & All & $\mathrm{DI}, \mathrm{D}$ & Re-evaluated AR & 210 & Underregistration of $10 \%$ \\
\hline $\begin{array}{l}\text { Malla, A. } \\
\text { and }\end{array}$ & 1983 & 1974-1978 & $\begin{array}{l}\text { Newfoundland } \\
\text { (Canada) }\end{array}$ & All & $\mathrm{S}, \mathrm{CO}$ & $\begin{array}{l}\text { Re-evaluated DC } \\
\text { and AR }\end{array}$ & 104 & १ी 12\% under-reporting. \\
\hline
\end{tabular}
Hoenig, J

[16]

\begin{tabular}{|c|c|c|c|c|c|c|c|}
\hline & & & & & & & $\begin{array}{l}\text { Study suicide rate of } 4.25 / \\
100,000 \text { suicides per year, } \\
\text { compared to the official } \\
\text { rate of } 3.8 / 100,000 \text { per year. }\end{array}$ \\
\hline $\begin{array}{l}\text { Clarke- } \\
\text { Finnegan, } \\
\text { M. and } \\
\text { Fahy, T. J }\end{array}$ & 1983 & 1978 & Galway, Ireland & All & $\begin{array}{l}\text { All deaths } \\
\text { except natural } \\
\text { deaths and } \\
\text { TD }\end{array}$ & Re-evaluated AR 410 & १ฯ 126\% under-reporting. \\
\hline
\end{tabular}

TD

[15]

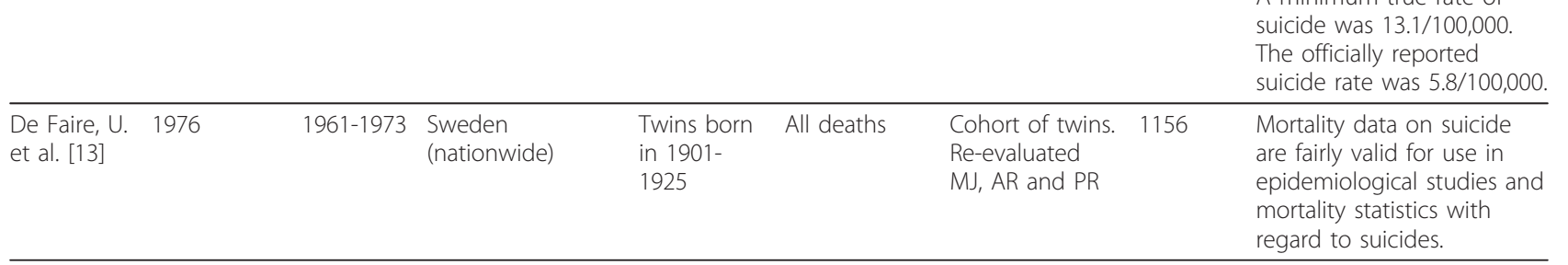

\begin{tabular}{|c|c|c|c|c|c|c|c|}
\hline $\begin{array}{l}\text { McCarthy, } \\
\text { P. D. and } \\
\text { Walsh, D } \\
{[11]}\end{array}$ & 1975 & 1964-1968 & Dublin (Ireland) & All & $\begin{array}{l}\text { All deaths } \\
\text { investigated } \\
\text { by Dublin city } \\
\text { and county } \\
\text { coroners }\end{array}$ & $\begin{array}{l}\text { Re-evaluated CR, } 210 \\
\text { MJ }\end{array}$ & १ी 279\% under-reporting. \\
\hline
\end{tabular}

Official suicide rate of 1.4 per 100,000 and study-rate of 5.3 per 100,000 .

\begin{tabular}{|c|c|c|c|c|c|c|c|c|}
\hline $\begin{array}{l}\text { Ovenstone, } \\
\text { I.M.K [9] }\end{array}$ & 1973 & $\begin{array}{l}\text { Oct.1969- } \\
\text { March } \\
1971\end{array}$ & $\begin{array}{l}\text { Edinburgh } \\
\text { (Scotland) }\end{array}$ & All & $\begin{array}{l}\text { S, A, U and } \\
\text { gas } \\
\text { poisonings }\end{array}$ & Re-evaluated MJ & 214 & $\begin{array}{l}\text { Suicide rate was } \\
\text { underestimated by } \\
\text { approximately } 50 \% \text { by the } \\
\text { Crown Counsel. }\end{array}$ \\
\hline
\end{tabular}


Table 1 Characteristics of the included studies by type of study and publication year (Continued)

\begin{tabular}{|c|c|c|c|c|c|c|c|c|}
\hline & & & & & & & & $\begin{array}{l}\text { The Crown counsel under- } \\
\text { reporting suicide by } 40.6 \% \\
\text { and the Scottish Registrar } \\
\text { General } 32 \% \text {. }\end{array}$ \\
\hline $\begin{array}{l}\text { Litman, R.E. } \\
\text { et al. [8] }\end{array}$ & 1963 & 1959-1960 & $\begin{array}{l}\text { Los Angeles } \\
\text { County }\end{array}$ & All & $\begin{array}{l}\text { Equivocal } \\
\text { suicides }\end{array}$ & $\begin{array}{l}\text { Re-evaluated PR, } \\
\text { AR and } \\
\text { interviewed } \\
\text { survivors of the } \\
\text { deceased. }\end{array}$ & 100 & Significant underreporting. \\
\hline \multicolumn{9}{|c|}{ Combined re-evaluation and registry studies } \\
\hline \multirow[t]{2}{*}{$\begin{array}{l}\text { Cantor, C. } \\
\text { et al. [32] }\end{array}$} & 2001 & 1990-1995 & $\begin{array}{l}\text { Queensland, } \\
\text { Australia }\end{array}$ & All & $\begin{array}{l}\text { Suicide } \\
\text { beyond no } \\
\text { reasonable } \\
\text { doubt, } \\
\text { probable and } \\
\text { possible } \\
\text { suicide }\end{array}$ & $\begin{array}{l}\text { Registry study } \\
\text { and re- } \\
\text { evaluated PR } \\
\text { and AR }\end{array}$ & 2728 & $\begin{array}{l}5.5 \% \text { more suicides } \\
\text { registered in QSR than the } \\
\text { official ABS count for the } \\
\text { period. }\end{array}$ \\
\hline & & & & & & & & $\begin{array}{l}141 \text { of the deaths coded as } \\
\text { probable/possible suicides } \\
(675) \text { by the QSR were } \\
\text { rejected as being deaths } \\
\text { other than suicide - usually } \\
\text { accidental overdoses. }\end{array}$ \\
\hline $\begin{array}{l}\text { Thorslund, J } \\
\text { et al. [21] }\end{array}$ & 1989 & 1977-1986 & Greenland & All & $\mathrm{S}, \mathrm{CO}$ & $\begin{array}{l}\text { Registry study } \\
\text { and Re- } \\
\text { evaluated DC } \\
\text { and PR }\end{array}$ & 442 & $\begin{array}{l}\text { The official statistics are } \\
\text { generally reliable. }\end{array}$ \\
\hline \multicolumn{9}{|c|}{ Registry studies } \\
\hline $\begin{array}{l}\text { Elnour, A.A. } \\
\text { and } \\
\text { Harrison, J } \\
{[38]}\end{array}$ & 2009 & $\begin{array}{l}\text { July } 2000 \\
\text { - the end } \\
\text { of } 2005\end{array}$ & $\begin{array}{l}\text { Australia } \\
\text { (nationwide) }\end{array}$ & All & $S$ & Registry study & 12786 & About $8 \%$ underestimation \\
\hline $\begin{array}{l}\text { Lahti, R.A. } \\
\text { and Vuori, } \\
\text { E [35] }\end{array}$ & 2003 & 1997 & $\begin{array}{l}\text { Finland } \\
\text { (nationwide) }\end{array}$ & All & DPD; $A, S, U$ & Registry study & 500 & $\begin{array}{l}\text { Fairly good agreement. } \\
98.5 \% \text { agreement }\end{array}$ \\
\hline $\begin{array}{l}\text { Lindeman } \\
\text { S.M. et al. } \\
{[27]}\end{array}$ & 1995 & 1986-1991 & $\begin{array}{l}\text { Finland } \\
\text { (nationwide) }\end{array}$ & All & $S, U, A^{* * *}$ & Registry study & 17412 & $\begin{array}{l}\text { Reliable enough. 97\% } \\
\text { coverage. }\end{array}$ \\
\hline \multirow{2}{*}{$\begin{array}{l}\text { Van de } \\
\text { Voorde, } \mathrm{H} \text {. } \\
\text { et al. [25] }\end{array}$} & 1993 & $1981-1984$ & Leuven (Belgium) & All & S & Registry study & 323 & $\begin{array}{l}\text { Incidence reporting bias of } \\
4-8 \% \text {. }\end{array}$ \\
\hline & & & & & & & & $\begin{array}{l}311 \text { suicides found in one } \\
\text { registry (PPO) and } 323 \\
\text { suicides in the other } \\
\text { registry (NIS). }\end{array}$ \\
\hline $\begin{array}{l}\text { Gary Hlady, } \\
\text { W. and } \\
\text { Middaugh, } \\
\text { J.P [19] }\end{array}$ & 1988 & 1983-1984 & Alaska & All & $S$ & Registry study & 195 & $\begin{array}{l}\text { Severely under-recorded } \\
\text { suicides. }\end{array}$ \\
\hline \multirow{2}{*}{$\begin{array}{l}\text { Marshall, D. } \\
\text { L. and } \\
\text { Soule, S } \\
{[18]}\end{array}$} & 1988 & 1979-1984 & $\begin{array}{l}49 \text { predominantly } \\
\text { Native villages in } \\
\text { southwest Alaska }\end{array}$ & All & $V D ; S, A, U, H$ & Registry study & 220 & ११405\% under-reporting. \\
\hline & & & & & & & & $\begin{array}{l}\text { Native suicide rate } 36.9 \text { / } \\
100,000(n=38) \text {, compared } \\
\text { to official suicide rate } 7.3 / \\
100,000(n=5) \text {. }\end{array}$ \\
\hline \multicolumn{9}{|c|}{ "Other re-evaluation studies" } \\
\hline $\begin{array}{l}\text { Joseph, A. } \\
\text { et al. [34] }\end{array}$ & 2003 & 1994-1999 & $\begin{array}{l}\text { Kaniyambadi } \\
\text { region, southern } \\
\text { India }\end{array}$ & All & - & Verbal autopsies & - & $\begin{array}{l}\text { Mean suicide rate was 95.2/ } \\
100,000 \text { (range } 83.7-106.3 \text { / } \\
100,000 \text { ) - nine times the } \\
\text { national average }\end{array}$ \\
\hline
\end{tabular}


Table 1 Characteristics of the included studies by type of study and publication year (Continued)

\begin{tabular}{|c|c|c|c|c|c|c|c|c|}
\hline $\begin{array}{l}\text { Warshauer, } \\
\text { M.E. and } \\
\text { Monk, M } \\
{[14]}\end{array}$ & 1978 & 1968-1970 & $\begin{array}{l}\text { An area of New } \\
\text { York City; East } \\
\text { Harlem in } \\
\text { Manhattan and } \\
\text { three districts in } \\
\text { the South Bronx. } \\
\text { (USA) }\end{array}$ & All & $\begin{array}{l}\text { S, "assigned" } \\
\text { suicide }\end{array}$ & $\begin{array}{l}\text { Comparing } \\
\text { published } \\
\text { Health } \\
\text { Department } \\
\text { suicide rates } \\
\text { with medical } \\
\text { examiner } \\
\text { records }\end{array}$ & - & $\begin{array}{l}\text { Suicides among the black } \\
\text { population were } \\
\text { underestimated by } 80 \% \text { and } \\
\text { those among whites by } \\
42 \% \text {. Suicide rates were } \\
\text { greatly affected by the } \\
\text { change in revision of the } \\
\text { ICD. }\end{array}$ \\
\hline $\begin{array}{l}\text { Atkinson, } \\
\text { M.W. et al. } \\
{[10]}\end{array}$ & 1975 & - & $\begin{array}{l}\text { England and } \\
\text { Denmark } \\
\text { (nationwide) }\end{array}$ & All & $\begin{array}{l}\text { Probable } \\
\text { suicide }\end{array}$ & $\begin{array}{l}\text { Compared } \\
\text { suicide } \\
\text { ascertainment } \\
\text { procedure } \\
\text { between } \\
\text { Denmark and } \\
\text { England. }\end{array}$ & 40 & $\begin{array}{l}\text { Danes consistently report } \\
\text { more suicides than do the } \\
\text { English coroners }\end{array}$ \\
\hline $\begin{array}{l}\text { Ross, } \mathrm{O} . \\
\text { and } \\
\text { Kreitman, N } \\
{[12]}\end{array}$ & 1975 & - & $\begin{array}{l}\text { England, Wales, } \\
\text { Scotland } \\
\text { (nationwide) }\end{array}$ & All & $S, U, O V$ & $\begin{array}{l}\text { A two-way } \\
\text { exchange of } \\
\text { case records }\end{array}$ & 264 & $\begin{array}{l}\text { The results strongly suggest } \\
\text { that the two sets of officials } \\
\text { share roughly the same } \\
\text { criteria. }\end{array}$ \\
\hline
\end{tabular}

$A$ accident, $A D$ all deaths, $A R$ autopsy-/forensic-/post-mortem reports, $C O$ controversial/unclear/no cause of death reported, $C R$ coroner/medical examination records, $D$ drowning, $D C$ death certificates/report, $D /$ deaths from intoxication, DPD drug poisoning deaths, $H$ : homicide, $J P$ judicial proceedings, $M J$ medical- $/$ hospital journal/-report, $N D$ natural death where insufficient medical information exists to permit the issuing of a death certificate stating that the cause of death was a natural one. OV open verdict, PR police reports, $S$ suicide, $S N$ suicide note, TE testimonies of relatives and witnesses, TD traffic deaths, TR toxicology reports, $U$ undetermined, $V D$ violent deaths, $A^{*}$ accidents by handgun, overdose, asphyxia, drowning and falling. $A^{* *}$ road traffic accidents, fires in domestic dwellings and drownings were excluded. $A^{* * *}$ motor vehicle accident, drowning, poisoning

I The authors have calculated the under-reporting: Found 18 "new suicides", total 1219 suicide and suicide rate 13.76/100,000 in Sweden in 1999 (data from Socialstyrelsen)

ๆๆ ("study suicide rate" - official suicide rate)/official suicide rate

reporting of $0-10 \%$. Of the 31 studies from the 46 -year period, 52\% (16 of 31 studies) found more than 10\% under-reporting, and 39\% (12 of 31 studies) found more than $30 \%$ under-reporting or poor suicide statistics. A summary of the conclusins of the included studies are presented in Table 2.

\section{Analysis of methodological quality}

When summarizing the quality scores three studies $[24,33,36]$ got a sum score $\geq 15$, representing good quality. One of these studies concluded fairly reliable suicide statistics, one $0-10 \%$ under-reporting and one concluded $11-30 \%$ under-reporting. Twenty-one studies got a sum score 10-14 [8,9,11-13,15-17,20-23,27-32,35,37,38] and seven studies sum score $\leq 9[10,14,18,19,25,26,34]$. Five of the studies with quality sum score $\leq 9$ concluded $>$ $30 \%$ under-reporting or poor suicide statistics, one concluded $0-10 \%$ under-reporting. The quality sum score and the conclusions of the studies are presented in Table 3

\section{Discussion}

\section{Summary of main results}

The main finding was that few studies on the reliability of suicide statistics have been done in recent years, and few countries have been covered. There were only two studies from Asia and none from Africa, where a large proportion of the global population resides. Thirteen of the 31 studies included in this review concluded with fairly reliable suicide statistics or under-reporting of 0 -
$10 \%$. Of the 31 studies from the 46 -year period, $52 \%$ found more than 10\% under-reporting, and 39\% found more than $30 \%$ under-reporting or poor suicide statistics. Eleven studies evaluated a nationwide sample, and only two studies compared data from two or more countries. Only three studies got a good quality sum score. It is a trend that studies with high quality sum score concluded with fairly reliable suicide statistics or under-reporting of $0-10 \%$, while studies with poorer quality sum score tends to conclude with more than $30 \%$ under-reporting or poor suicide statistics, but too few studies are done to make an absolute conclusion. We have put most emphasis on the studies with the best methodological quality. These studies support our main findings. We cannot make any conclusions about the reliability of suicide statistics based only on the lack of research. Theoretically, the reliability might be good in spite of the lack of studies. In countries with official suicide rates close to zero, one might argue that the reliability was good. It is important to study the reliability of suicide statistics, and since the data are very different in the various countries, we find it of importance to study both the validity and reliability. As there are few studies, and about half of them concluded with underreporting of suicide, we think that our main finding, that the reliability of suicide statistics is questionable and calls for more studies, is fear.

Reliability does not necessarily imply validity. A reliable measure is measuring something consistently, but it 
Table 2 Brief summary table

\begin{tabular}{llll}
\hline Conclusions & Total number of studies $(\mathbf{n})$ & Re-evaluation studies & Registry studies \\
\hline $0-10 \%$ under-reporting & 7 & 4 & 3 \\
\hline Fairly reliable suicide statistics & 6 & 3 & 3 \\
\hline $11-30 \%$ under-reporting & 4 & 4 & 0 \\
\hline$>30 \%$ under-reporting & 10 & 9 & 1 \\
\hline Poor suicide statistics & 2 & 1 & 1 \\
\hline
\end{tabular}

The two combined re-evaluation and registry studies are in this table registered as registry studies.

The two studies were they exchanged data between countries (Ross O. et al. [12] and Atkinson M.W. et al. [10]) are not included in this table.

may not measure what it claims to do. A thorough forensic and psychological autopsy may be the most valid method to determine the cause of death, e.g. suicide. If the statistics consistently underreported suicide, the methods could be reliable enough to justify multivariate analyses of determinants and multivariate evaluations of interventions. In the present study, the causes of death were assessed by official statistics and the researchers had the intention of measuring the same phenomenon. Accordingly, we consider that comparing official suicide statistics and external assessments reflects both reliability and validity.

Studying the reliability of suicide statistics is a complex task. First, some suicides might have been missed in the administrative processes of national mortality statistics. Second, in some cases, determining the manner of death (i.e., suicide, accident, undetermined/open verdict, or natural death) requires subjective interpretation of the intention of the deceased. Different methodologies used in the included studies need to be considered, including the main difference between re-evaluation and registry studies, the variations in the cause and manner of deaths studied, the quality of the compared registers, the competence of the re-evaluators, the number of reevaluations of each case and the information the re-evaluations are based on (i.e., death certificates, police reports, autopsy reports, etc.). One can imagine that a greater number of suicides could be found by also examining undetermined deaths/open verdicts and accidents. Some studies (statistical studies) have studied the under-reporting of suicide by comparing the suicide rate with the rate of deaths of undetermined intent [39,40], and in recent years, the UK has added injury/poisoning of undetermined intent and sequelae of intentional selfharm/event of undetermined intent to the official suicide rate, in the belief it will provide a more reliable suicide rate [41]. We excluded statistical studies in this review article, but in a national and longitudinal perspective these studies are important for indicating reliability of suicide statistics, and further, effects of suicide prevention. Studies included in this review were published in many different countries between 1963 and 2009. Hence, different editions of the ICD are used in these studies, which may also affect the results to a certain extent [42].

\section{Strengths and limitations}

Some limitations of the present study should be considered. The search strategy, including literature search and reference list screening, was developed by one of the authors, and this search strategy may not have captured all relevant studies. Manually searching reference lists located 33 further studies not captured in the database searches. The selection of keywords and MeSH terms that were used may not have covered all published articles on the reliability of suicide statistics. The choice of databases also needs to be considered. The five selected databases may not have indexed all potential studies, and some relevant studies may not have been included. Medline is the largest component of PubMed, and both databases were selected in the present study because they do not have the same $\mathrm{MeSH}$ terms, and therefore more studies were found searching both http://www.nlm.nih.gov/pubs/factsheets/dif_med_pub.html. In retrospect, it is conceivable that using only one of these databases might have saved time, and we

Table 3 Summary quality sum score

\begin{tabular}{llll}
\hline Conclusions & Quality sum score $\mathbf{5 9}$ & Quality sum score $\mathbf{1 0 - 1 4}$ & Quality sum score $\mathbf{\geq} \mathbf{1 5}$ \\
\hline 0-10\% under-reporting & 1 & 5 & 1 \\
\hline Fairly reliable suicide statistics & 0 & 5 & 1 \\
\hline $11-30 \%$ under-reporting & 0 & 3 & 1 \\
\hline$>30 \%$ under-reporting & 4 & 6 & 0 \\
\hline Poor suicide statistics & 1 & 1 & 0 \\
\hline
\end{tabular}

The two studies were they exchanged data between countries (Ross O. et al. [12] and Atkinson M.W. et al. [10]) are not included in this table. 
might have found more studies by selecting a different database or manually searched relevant journals. It is possible that our search did not identify all of the relevant original studies [43]; for example, the publications of national statistics bureaus are not indexed in the databases, but we are confident that our research strategy has been good enough to identify the majority of the relevant original studies. Even though we may have missed some studies, we find it unlikely that this would have changed our main conclusions. For practical reasons, only published studies were sourced, but it seems unlikely that publication status would be a source of bias in the present study.

One strength of this review is that related studies and all reference lists of the included studies were screened, minimizing the number of potentially missed studies. Another strength is that the three authors independently screened all abstracts and full-text articles, minimizing the chance of a relevant study being excluded.

\section{Future studies}

The fact that few studies have been published in recent years, makes further studies clearly needed, particulary nationwide studies, studies in countries with low suicide rates and other under-investigated countries, and studies including comparisons between countries.

\section{Conclusion}

There are only few studies on the reliability of suicide statistics, and based on those studies, we cannot draw firm conclusions about the reliability of existing suicide statistics. Few studies have been published in recent years. Nationwide studies in particular are lacking, and only two studies compared data between countries.

This systematic review conforms to the PRISMA statement [44].

\section{Additional material}

Additional file 1: Search terms

\section{Acknowledgements}

This study was supported by a grant from the South Eastern Norway Regional Health Authority.

\section{Author details}

'Department of Acute Medicine, Oslo University Hospital Ullevaal, PO Box 4956, Nydalen NO-0424 Oslo, Norway. ${ }^{2}$ Department of Behavioural Sciences in Medicine, Institute of Basic Medical Sciences, Faculty of Medicine, University of Oslo, PO Box 1111, Blindern NO-0317 Oslo, Norway.

\section{Authors' contributions}

All authors contributed equally to the conception and design of the study, the selection of studies, and to the final version of the manuscript. IMT developed the search strategy, interpreted the data and drafted the manuscript. All authors read and approved the final manuscript.

\section{Competing interests}

The authors declare that they have no competing interests.

Received: 21 March 2011 Accepted: 14 February 2012

Published: 14 February 2012

\section{References}

1. World Health Organization: Mental Health. Policy and Services Geneva; 2011.

2. World Health Organization: World Health Report 2003: Shaping the Future Geneva; 2003.

3. Welch SS: A review of the literature on the epidemiology of parasuicide in the general population. Psychiatr Serv 2001, 52:368-375.

4. Schmidtke A, Bille-Brahe U, DeLeo D, Kerkhof A, Bjerke T, Crepet P, Haring C, Hawton K, Lonnqvist J, Michel K, et al: Attempted suicide in Europe: rates, trends and sociodemographic characteristics of suicide attempters during the period 1989-1992. Results of the WHO/EURO multicentre study on parasuicide. Acta Psychiatr Scand 1996, 93:327-338.

5. World Health Organization: Country Reports and Charts 2010 [http://www. who.int/mental_health/prevention/suicide/country_reports/en/index.html].

6. Johansson LA, Pavillon G, Anderson R, Glenn D, Griffiths C, Hoyert D, Jackson G, Notzon FS, Rooney C, Rosenberg HM, et al: Counting the dead and what they died of. Bull World Health Organ 2006, 84:254.

7. Pearson-Nelson BJ, Raffalovich LE, Bjarnason T: The effects of changes in the world health organization's international classification of diseases on suicide rates in 71 countries, 1950-1999. Suicide Life Threat Behav 2004, 34:328-336.

8. Litman RE, Curphey TJ, Shneidman ES, Farberow NL, Tabachnick N: Investigations of equivocal suicides. JAMA 1963, 184:924-929.

9. Ovenstone IM: A psychiatric approach to the diagnosis of suicide and its effect upon the Edinburgh statistics. Br J Psychiatry 1973, 123:15-21.

10. Atkinson MW, Kessel N, Dalgaard JB: The comparability of suicide rates. $\mathrm{Br}$ J Psychiatry 1975, 127:247-256.

11. McCarthy PD, Walsh D: Suicide in Dublin: I. The under-reporting of suicide and the consequences for national statistics. Br J Psychiatry 1975, 126:301-308.

12. Ross $\mathrm{O}$, Kreitman $\mathrm{N}$ : A further investigation of differences in the suicide rates of England and Wales and of Scotland. Br J Psychiatry 1975, 127:575-582

13. de Faire U, Friberg $L$, Lorich U, Lundman T: A validation of cause-of-death certification in 1,156 deaths. Acta Med Scand 1976, 200:223-228.

14. Warshauer ME, Monk M: Problems in suicide statistics for whites and blacks. Am J Public Health 1978, 68:383-388.

15. Clarke-Finnegan M, Fahy TJ: Suicide rates in Ireland. Psychol Med 1983, 13:385-391.

16. Malla A, Hoenig J: Differences in suicide rates: an examination of underreporting. Can J Psychiatry 1983, 28:291-293.

17. Ekeberg $O$, Jacobsen $D$, Enger $E$, Frederichsen $P$, Holan L: The reliability of suicide statistics in Norway. Tidsskr Nor Laegeforen 1985, 105:123-127.

18. Marshall DL, Soule S: Accidental deaths and suicides in southwest Alaska: actual versus official numbers. Alaska Med 1988, 30:45-52.

19. Hlady WG, Middaugh JP: The underrecording of suicides in state and national records, Alaska, 1983-1984. Suicide Life Threat Behav 1988, 18:237-244.

20. Huusko R, Hirvonen J: The problem of determining the manner of death as suicide or accident in borderline cases. Z Rechtsmed 1988, 100:207-213.

21. Thorslund J, Misfeldt J: On suicide statistics. Arctic Med Res 1989, 48:124-130.

22. Walsh D, Cullen A, Cullivan R, O'Donnell B: Do statistics lie? Suicide in Kildare- and in Ireland. Psychol Med 1990, 20:867-871.

23. Rodriguez-Pulido F, Sierra A, Gracia R, Doreste J, Delgado S, GonzalezRivera JL: Suicide in the Canary Islands, 1977-1983. Acta Psychiatr Scand 1991, 84:520-523.

24. Allebeck P, Allgulander C, Henningsohn L, Jakobsson SW: Causes of death in a cohort of 50,465 young men-validity of recorded suicide as underlying cause of death. Scand J Soc Med 1991, 19:242-247.

25. van de Voorde $H$, Hooft P, Mulkers U: On the influence of data source in aggregated data studies: a comparative study of suicide information based on death certificates and judicial files. J Epidemiol Community Health 1993, 47:73-75.

26. Scott KW: Suicide in Wolverhampton (1976-1990). Med Sci Law 1994, 34:99-105 
27. Lindeman SM, Hirvonen Jl, Hakko HH, Lonnqvist JK: Use of the national register of medico-legal autopsies in epidemiological suicide research. Int J Legal Med 1995, 107:306-309.

28. Connolly JF, Cullen A: Under-reporting of suicide in an Irish county. Crisis 1995, 16:34-38.

29. O'Donnell I, Farmer R: The limitations of official suicide statistics. Br J Psychiatry 1995, 166:458-461.

30. Ohberg A, Lonnqvist J: Suicides hidden among undetermined deaths. Acta Psychiatr Scand 1998, 98:214-218.

31. Sampson HH, Rutty GN: Under-reporting of suicide in South Yorkshire (West): a retrospective study of suicide and open verdicts returned by HM Coroner, 1992-1997. J Clin Forensic Med 1999, 6:72-76.

32. Cantor C, MCTaggart P, De LD: Misclassification of suicide-the contribution of opiates. Psychopathology 2001, 34:140-146.

33. Ahlm K, Eriksson A, Lekander T, Bjornstig U: All traffic related deaths are not 'fatalities' -analysis of the official Swedish statistics of traffic accident fatalities in 1999. Lakartidningen 2001, 98:2016-2022.

34. Joseph A, Abraham S, Muliyil JP, George K, Prasad J, Minz S, Abraham VJ, Jacob KS: Evaluation of suicide rates in rural India using verbal autopsies, 1994-9. BMJ 2003, 326:1121-1122

35. Lahti RA, Vuori E: Fatal drug poisonings: medico-legal reports and mortality statistics. Forensic Sci Int 2003, 136:35-46.

36. Carr JR, Hoge CW, Gardner J, Potter R: Suicide surveillance in the U.S. Military-reporting and classification biases in rate calculations. Suicide Life Threat Behav 2004, 34:233-241.

37. Lu TH, Sun SM, Huang SM, Lin JJ: Mind your manners: quality of manner of death certification among medical examiners and coroners in Taiwan. Am J Forensic Med Pathol 2006, 27:352-354.

38. Elnour $A A$, Harrison J: Suicide decline in Australia: where did the cases go? Aust N Z J Public Health 2009, 33:67-69.

39. Kelleher MJ, Corcoran P, Keeley HS, Dennehy J, O’Donnell I: Improving procedures for recording suicide statistics. Ir Med J 1996, 89:14-15.

40. Speechley M, Stavraky KM: The adequacy of suicide statistics for use in epidemiology and public health. Can J Public Health 1991, 82:38-42.

41. Office for National Statistics: Suicide Rates in the United Kingdom, 2000-2009 Newport; 2011.

42. Jansson B, Johansson LA, Rosen M, Svanstrom L: National adaptations of the ICD rules for classification-a problem in the evaluation of cause-ofdeath trends. J Clin Epidemiol 1997, 50:367-375.

43. Dickersin K, Scherer R, Lefebvre C: Identifying relevant studies for systematic reviews. BMJ 1994, 309:1286-1291.

44. Moher D, Liberati A, Tetzlaff J, Altman DG: Preferred reporting items for systematic reviews and meta-analyses: the PRISMA statement. Int I Surg 2010, 8:336-341.

\section{Pre-publication history}

The pre-publication history for this paper can be accessed here: http://www.biomedcentral.com/1471-244X/12/9/prepub

doi:10.1186/1471-244X-12-9

Cite this article as: Tøllefsen et al:: The reliability of suicide statistics: a systematic review. BMC Psychiatry 2012 12:9.

\section{Submit your next manuscript to BioMed Central and take full advantage of:}

- Convenient online submission

- Thorough peer review

- No space constraints or color figure charges

- Immediate publication on acceptance

- Inclusion in PubMed, CAS, Scopus and Google Scholar

- Research which is freely available for redistribution

Submit your manuscript at www.biomedcentral.com/submit
Biomed Central 